\title{
Functional Magnetic Resonance Imaging Reveals Brain Regions Mediating the Response to Resistive Expiratory Loads in Humans
}

\author{
David Gozal, ${ }^{\star}$ Omid Omidvar, ${ }^{\star}$ Konrad A. T. Kirlew, ${ }^{\ddagger}$ Gasser M. Hathout, ${ }^{\ddagger}$ Robert B. Lufkin, ${ }^{\ddagger}$ and Ronald M. Harper ${ }^{\star}$ \\ Departments of $*$ Neurobiology and ${ }^{\ddagger}$ Radiology, University of California at Los Angeles School of Medicine, Los Angeles, California \\ 90095; and ${ }^{\S}$ Constance S. Kaufman Pedratric Pulmonary Research Laboratory, Departments of Pediatrics and Physiology, University of \\ Tulane School of Medicine, New Orleans, Louisiana 70112
}

\begin{abstract}
Obstructive lung disease is the most common form of respiratory disturbance. However, the location of brain structures underlying the ventilatory response to resistive expiratory loads is unknown in humans. To study this issue, midsagittal magnetic resonance images were acquired in eight healthy volunteers before and after application of a moderate resistive expiratory load $\left(30 \mathrm{cmH}_{2} \mathrm{O} / \mathrm{liter} / \mathrm{s}\right)$, using functional magnetic resonance imaging (fMRI) strategies (1.5-T magnetic resonance; repetition time: $72 \mathrm{~ms}$; echo time: $45 \mathrm{~ms}$; flip angle: $30^{\circ}$; field of view: $26 \mathrm{~cm}$; slice thickness: $5 \mathrm{~mm} ; 128 \times 256 \times 1$ number of excitations). Digital image subtractions and region of interest analyses revealed significant increases in fMRI signal intensity in discrete areas of the ventral medulla, ventral and dorsal pontomedullary structures, basal forebrain, and cerebellum. Upon load withdrawal, a rapid fMRI signal off-transient occurred in all activated sites. Application of an identical load immediately after recovery from the initial stimulus resulted in smaller signal increases $(P<0.02)$. Prolongation of load duration was associated with progressive fMRI signal decrease across activated regions. In three additional subjects, the threshold for significant MRI signal increases was established at expiratory loads $\geq 15 \mathrm{cmH}_{2} \mathrm{O} /$ liter/s and was dose dependent with increasing loads. We conclude that resistive expiratory loads $\geq 15 \mathrm{cmH}_{2} \mathrm{O} /$ liter/s elicit regional activation of discrete brain locations in humans. (J. Clin. Invest. 1996. 97:47-53.) Key words: control of breathing • brain mapping • resistive load $\bullet$ functional magnetic resonance imaging
\end{abstract}

\section{Introduction}

Appropriate ventilatory responses to bronchoconstriction and airflow obstruction are essential for maintenance of gas homeostasis in pulmonary disease. For example, perception of

Portions of this work have been presented at the 24th Society for Neuroscience Annual Meeting and have been published in abstract form (1994. Soc. Neurosci. Abstr. 20:A563.6).

Address correspondence to David Gozal, M.D., Section of Pediatric Pulmonology, Department of Pediatrics, SL-37, University of Tulane School of Medicine, 1430 Tulane Avenue, New Orleans, LA 70112. Phone: 504-588-5601; FAX: 504-588-5490; E-mail: dgozal@ tmcpop.tmc.tulane.edu

Received for publication 10 July 1995 and accepted in revised form 22 September 1995.

J. Clin. Invest.

(C) The American Society for Clinical Investigation, Inc.

0021-9738/96/01/0047/07 \$2.00

Volume 97, Number 1, January 1996, 47-53 expiratory loads may be preserved in mild asthmatics (1), while such perceptions are diminished in a subset of asthmatic patients who are at increased risk for developing respiratory failure and sudden death (2).

Studies on central mechanisms underlying respiratory sensation and consequent voluntary or automatic ventilatory response to expiratory loads in humans have either used subjective techniques involving psychophysical studies (3), or evoked potentials (4). Such studies documented the importance of higher brain areas in breathing. Ramsay and colleagues (5) visualized primary motor-sensory cortical activation during volitional expiration using positron emission tomographic $(\mathrm{PET})^{1}$ techniques (5). Other brain regions, such as the supplementary motor area, lateral pre-motor area, thalamus, and cerebellum, also demonstrated significant activity enhancements (5). Such studies suggest that multiple brain structures are involved in the integrative response to respiratory stimuli. Identification of activated brain regions in humans after resistive loading should provide important insights into sensory, motor, and integrative components of ventilatory control mechanisms.

Functional magnetic resonance imaging (fMRI) procedures allow noninvasive visualization of human brain sites during application of specific stimuli or task paradigms (6-9). Such procedures demonstrate that regional neural activation of several brain structures occurs in response to enhanced central chemoreceptor drive elicited by changes in inspired carbon dioxide concentrations (10), as well as after application of inspiratory resistive loads (11). Of particular interest, brainstem loci underlying ventilatory responses to respiratory stimuli were identified for the first time in humans. Visualization of pontomedullary regions by fMRI is not marred by inherent reconstruction artifacts of PET, such that fMRI emerges as the most promising technique for assessing functional relationships of more caudal brain structures. Since increased expiratory resistance is a frequent clinical occurrence, we attempted to determine which brain regions, and in particular which brainstem regions, display enhanced activation to increased expiratory loads in healthy human subjects. In addition, we sought to establish the detection threshold of the fMRI procedure, the load dependency of the response, and the temporal characteristics of the fMRI signal to short, repeated, and sustained stimulus applications.

\section{Methods}

Subjects. 11 healthy volunteers (10 males and 1 female; aged 28-46 yr) were studied, after giving informed consent. The study was approved by the Institutional Review Board at both institutions.

Magnetic resonance $(M R)$ imaging. The imaging device consisted

1. Abbreviations used in this paper: fMRI, functional magnetic resonance imaging; MR, magnetic resonance; PET, positron emission tomography; ROI, region of interest. 
of a 1.5-T MR scanner (General Electric Signa System, Milwaukee, WI) with a standard head coil. To minimize subject and head motion during the scanning procedures, snug-fitting foam attachments were used. Routine T1-weighted coronal and sagittal scout images were performed to identify the planes for subsequent imaging.

Spoiled gradient acquisition in steady state images were acquired using the following scanning parameters: repetition time, $72 \mathrm{~ms}$; echo time, $45 \mathrm{~ms}$; flip angle, $30^{\circ} ; 128 \times 256$ matrix and 1 number of excitations; $26 \mathrm{~cm}$ field of view, with a 5 -mm slice thickness. The spatial resolution achieved was $10.3 \mathrm{~mm}^{3} /$ voxel. Images were acquired from a sagittal plane slightly off midline (Fig. $1 A$ ), during baseline and expiratory loading conditions. Since the imaging approach in this study allows only for monoplanar acquisition over time, the selected sagittal plane aimed to optimize imaging of regions contained within brainstem and basal forebrain at the expense of less reliable representation of cortical structures. Therefore, the latter regions were not specifically explored. Each scan required $\sim 9.5 \mathrm{~s}$.

Stability of the MR unit was verified with serial spoiled gradient acquisition in steady state imaging of a standard Daily Quality Assurance phantom (model 46-28297861; GE Medical Systems, Milwaukee, WI). 60 images were intermittently obtained over a period of 18 min, and signal drift was not observed. The average signal variation ranged from 0.18 to $0.34 \%$ in high and low signal intensity regions of the phantom, respectively.

Test protocol. Subjects were imaged while lying comfortably in the scanner and breathing via a mouthpiece through a two-way nonrebreather respiratory valve (Hans Rudolph, Kansas City, MO) with nose clips. The dead space of the system was $\sim 40 \mathrm{ml}$, and resistance to airflow was measured at $<0.012 \mathrm{cmH}_{2} \mathrm{O} /$ liter/min for flow rates ranging from 0 to 200 liter/min. 15 images were acquired during baseline, unloaded conditions. Eight subjects then breathed through a flow-resistive load or resistor applied to the expiratory limb of the nonrebreather valve, which consisted of a plastic tube of $4 \mathrm{~mm}$ in diameter and $100 \mathrm{~mm}$ in length, eliciting an approximately constant resistance of $30 \mathrm{cmH}_{2} \mathrm{O} / \mathrm{liter} / \mathrm{s}$ at flows between 5 and 30 liter/min. A second set of 15 images was obtained, after which the load was removed, and 60 additional images were consecutively acquired. This time interval was established previously to allow for return of fMRI signal in activated sites to stable baseline values (11). At this point, the load was reapplied, and 45 images were successively obtained.

To determine dose dependency of responses, we studied three additional subjects, and sets of 15 images were obtained at baseline and at increasing expiratory resistances of $0,5,10,15,30$, and 45 $\mathrm{cmH}_{2} \mathrm{O} /$ liter/s. One trial with a resistive load of $60 \mathrm{cmH}_{2} \mathrm{O} /$ liter/s elicited unacceptable motion artifacts, and further experiments with such loads were not pursued. Resting periods of $\sim 15-20$ min were allowed between resistor applications, which were introduced in random order.

Data analysis. Images from each subject were transferred to a Sun Sparc Station 2 (Sun Microsystems, Mountain View, CA). Each image collected during application of expiratory load was digitally subtracted from the corresponding image obtained during baseline conditions, using an image processing routine written in ANSI C. The resultant subtraction images were then averaged and subjected to ANOVA on a pixel-by-pixel basis. In addition, resultant gray-scale subtraction displays were pseudocolored with reference to a difference scale that corresponded to a $P<0.01$. Region of interest (ROI) analyses for signal intensity were performed for each image in regions determined from visual inspection of activation images. Defined ROI, ranging from 6 to $64 \mathrm{~mm}^{2}$ (6-72 pixels), were placed in every activated region, while avoiding visible vessels, inner table of skull, or cerebrospinal fluid-containing areas. Average pixel intensities from each ROI were obtained on all images from each subject to generate signal intensity versus time curves.
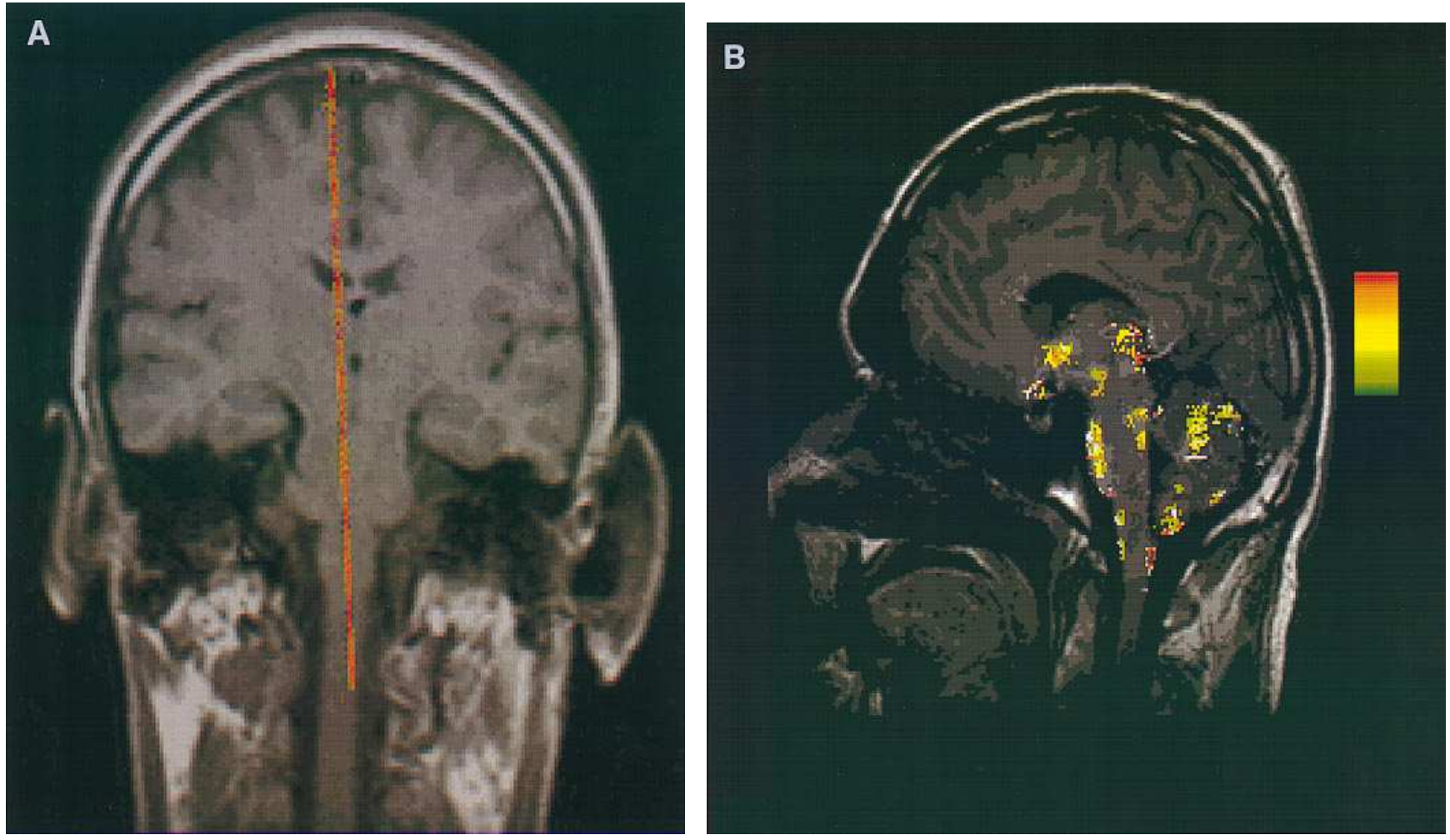

Figure 1. (A) Coronal T1 section in one subject. Red line indicates the fMRI sagittal imaging plane. (B) Midsagittal T1 image in one subject. Regions revealing significant fMRI signal enhancements during application of an expiratory resistive load in all eight subjects are shown on a pseudocolored scale in which each pixel was assigned either a transparent value when fMRI signal change did not achieve statistical significance, or a pseudocolored scale rank according to percent signal increase which was statistically significant. The pseudocolored scale comprises colors ranging from green (fMRI signal increase of $2 \%$ ) to red (maximal fMRI signal increase of $12 \%$ ). 
To normalize data across subjects, percentage frame-by-frame changes were calculated for each ROI in each subject by subtracting from the average ROI value of the initial 15 frames. In addition, ROI percent changes were averaged across subjects for each image, and ROI locations across different subjects were matched from individual anatomical landmarks for graphic purposes.

Values for baseline and initial and subsequent expiratory loaded conditions were then subjected to ANOVA procedures, followed by Tukey post-hoc tests, using BMDP statistical software (University of California, 1990, Berkeley, CA). A probability value of $<0.05$ was required to reach statistical significance.

\section{Results}

Significant increases in fMRI signal intensity occurred during expiratory loads in several regions which are shown in Fig. 1. In the sagittal plane, distinct ventral and dorsal medullary sites emerged, with the cuneatus and gracile nuclei activating in the dorsal region. A large portion of the ventral pons and discrete areas within the parabrachial pons and locus coeruleus displayed activation patterns. More rostrally, increased activity developed in the midline dorsal thalamus, basal forebrain, and interpeduncular nucleus. Signal enhancements also emerged in several areas within the cerebellum near the primary fissure on the dorsal surface and medial regions and in midline caudalventral sites (Fig. 1).

In three subjects exposed to resistive expiratory random load intensities, ROI analysis revealed significant MRI signal increases at loads $\geq 15 \mathrm{cmH}_{2} \mathrm{O}$ in all activated sites (Fig. 2). Dose-dependent responses emerged in all three subjects at all activated locations, appearing more pronounced in pontomedullary regions (Fig. 2).

Mean peak changes at various activated regions during initial $30 \mathrm{cmH}_{2} \mathrm{O}$ resistor application, as well as mean peak changes occurring during second load application, are shown in Table I. In general, a second load was associated with significant decreases in the magnitude of MR signal change at all ROI sites in which a significant signal response occurred.
Table I. Mean ( \pm SD) Peak Changes in MR Signal Intensity (Percent Change) in Various Regions of Interest upon Application of an Inspiratory Load

\begin{tabular}{lccc}
\hline \multicolumn{1}{c}{ Site } & I & II & $P$ value \\
\hline Ventral pons & $8.6 \pm 2.4$ & $5.2 \pm 2.1$ & $<0.01$ \\
Dorsal pons & $5.4 \pm 1.9$ & $4.9 \pm 2.2$ & NS \\
Ventral medulla & $4.7 \pm 1.3$ & $3.2 \pm 1.4$ & $<0.05$ \\
Dorsal medulla & $8.2 \pm 2.0$ & $6.2 \pm 1.7$ & $<0.05$ \\
Cerebral peduncle & $3.6 \pm 1.2$ & $2.7 \pm 1.1$ & $<0.05$ \\
Rostral cerebellum & $6.1 \pm 1.1$ & $4.2 \pm 0.9$ & $<0.01$ \\
Caudal cerebellum & $5.4 \pm 1.4$ & $3.7 \pm 1.1$ & $<0.01$ \\
Thalamus & $6.6 \pm 1.5$ & $4.6 \pm 1.3$ & $<0.01$ \\
Hypothalamus & $4.2 \pm 1.4$ & $3.5 \pm 0.9$ & $\mathrm{NS}$ \\
& & & \\
\hline
\end{tabular}

I and II, first and second application of inspiratory load.

Frame-by-frame signal analysis during load application revealed a uniform MR signal off-transient pattern across activated structures characterized by an immediate signal return to baseline upon discontinuation of the resistive stimulus (Figs. 3 and 4).

Sustained application of expiratory loads resulted in progressive MR signal decreases over time within all activated sites. Nadir fMRI signal values were within those measured before initiation of the resistive load. The onset of significant signal decreases occurred after $\sim 4-6$ min of continuous expiratory load application (range: 225-315 s). Both onset times and signal decrease rates over time were similar for all activated sites (Fig. 5).

\section{Discussion}

Enhanced fMRI signals occurred in discrete brain regions with application of resistive expiratory loads to eight healthy human subjects. The data provide initial information on the func-

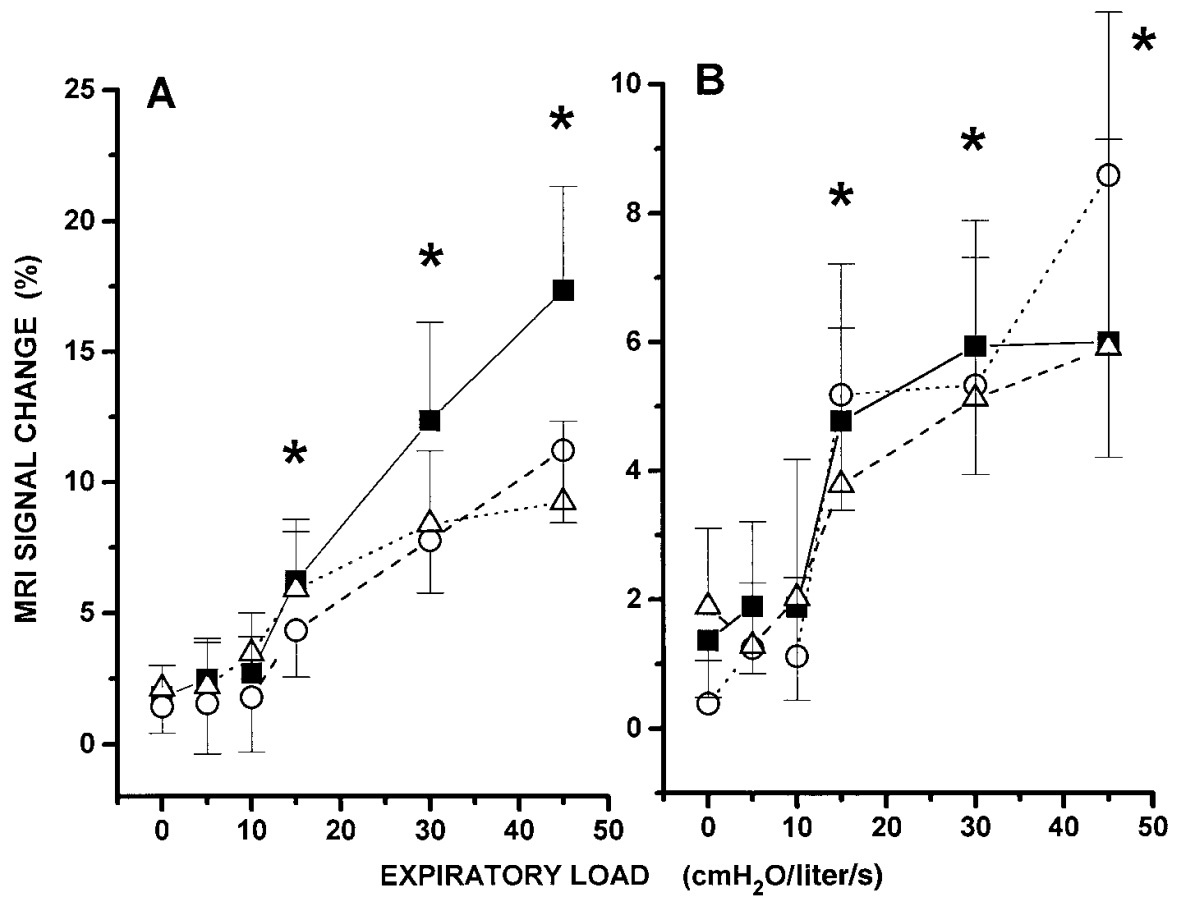

Figure 2. MR signal changes in three subjects in ventral pons $(A)$ and dorsal cerebellum $(B)$ after random application of expiratory loads ranging from 0 to $45 \mathrm{cmH}_{2} \mathrm{O}$. Each point represents mean $( \pm S D)$ of 15 images acquired during each load. $* P<$ 0.05 compared with unloaded conditions. 


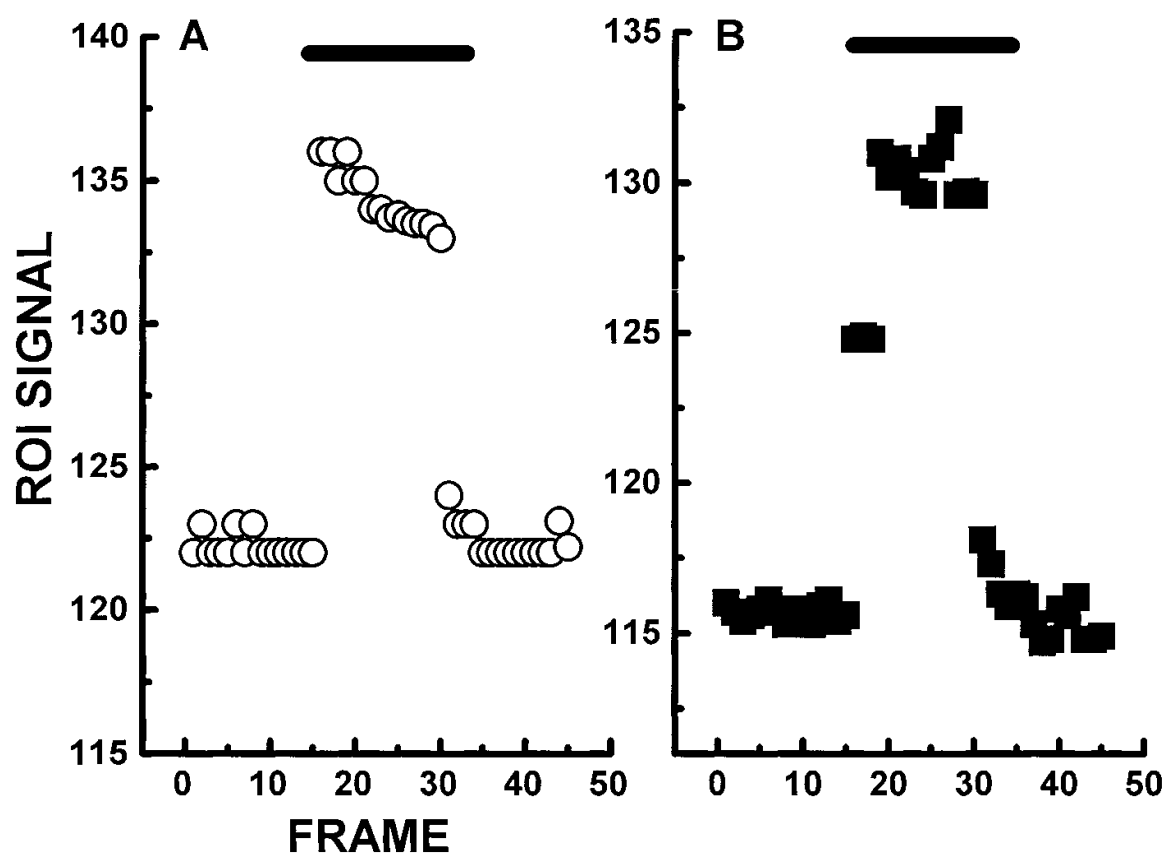

Figure 3. Frame-by-frame evolution of fMRI signal in a single subject, within ROI located in the ventral pons $(A)$ and dorsal cerebellum $(B)$, before, during, and after application of a $30 \mathrm{cmH}_{2} \mathrm{O}$ expiratory load. Note almost immediate signal return to baseline upon stimulus discontinuation. tional topography of the central nervous system response to imposed respiratory loads. Additional experiments in three subjects suggested that, within the limitations of the magnetic field strength, significant regional signal changes emerge only above an expiratory load threshold of $\geq 15 \mathrm{cmH}_{2} \mathrm{O}$. Furthermore, signals abruptly returned to baseline with stimulus cessation, and subsequent application of an identical respiratory load elicited diminished responses. Finally, sustained stimulus application was associated with gradual decreases in signal to initial baseline values; the declines began within 4 to 6 min of load application.

Marked alterations in regional cerebral blood flow and volume, neural tissue metabolic rates, and local oxygen extraction and utilization occur with neuronal discharge (12-16). Initial net decreases in regional deoxyhemoglobin are one of the early events of neural activation and can be promptly identified as signal increases by gradient echo and $\mathrm{T} 2 *$-weighted echoplanar MR imaging pulse sequences $(6-8,17)$. The regionalized decreases in deoxyhemoglobin, which have been confirmed by near-infrared spectroscopy (18), are postulated to result from excessive capillary blood flow recruitment within activated neural sites, without a concomitant parallel increase in oxygen tissue requirements, since the early phases of neuronal discharge are suggested to be primarily a nonoxidative process $(15,16)$. Such surplus of arterial blood flow would induce a decrease in the deoxyhemoglobin concentration of the venous capillary bed draining the activated site, which could be then used as an intrinsic MRI contrast agent by virtue of the different magnetic properties inherent to oxy- and deoxyhemoglobin moieties $(6,17)$. Indeed, blood oxygenation

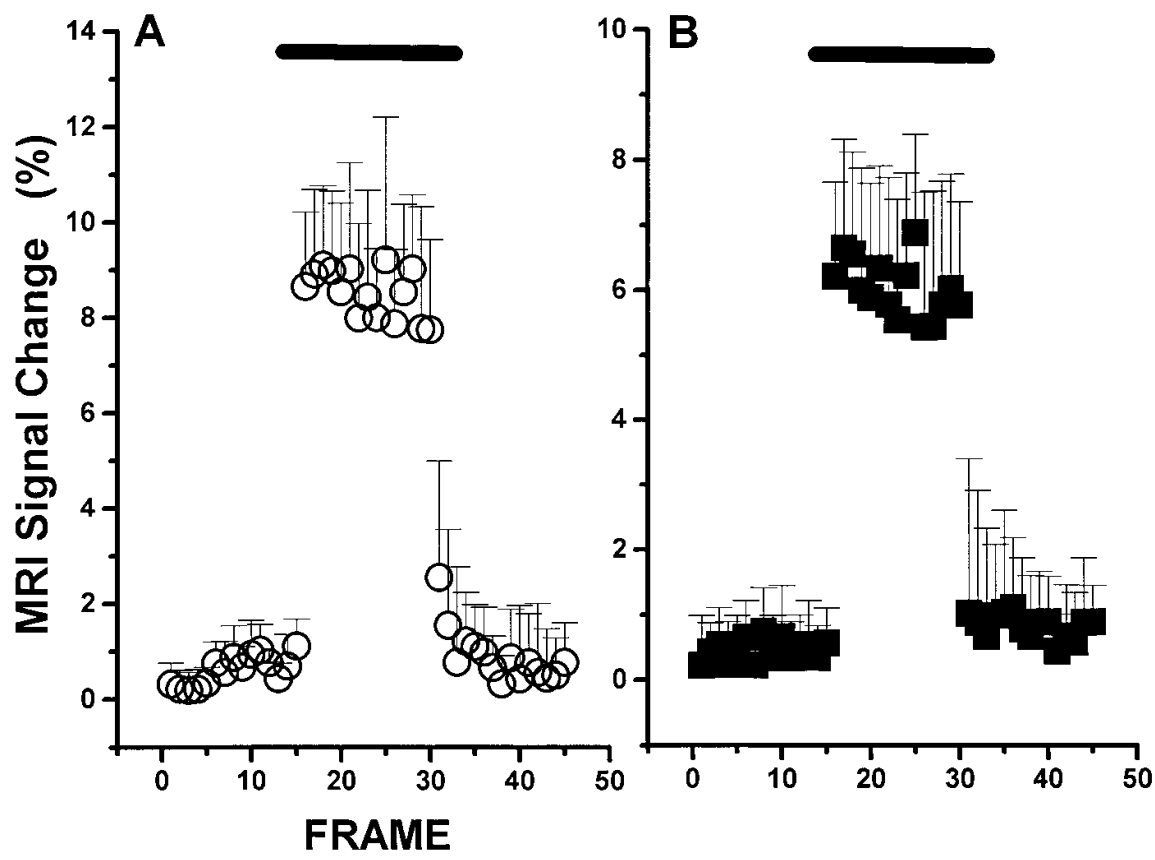

Figure 4. Mean $( \pm$ SD) frame-by-frame fMRI signal changes in eight subjects in the ventral pons $(A)$ and dorsal cerebellum $(B)$ before, during, and after application of a 30 $\mathrm{cmH}_{2} \mathrm{O}$ expiratory load. 


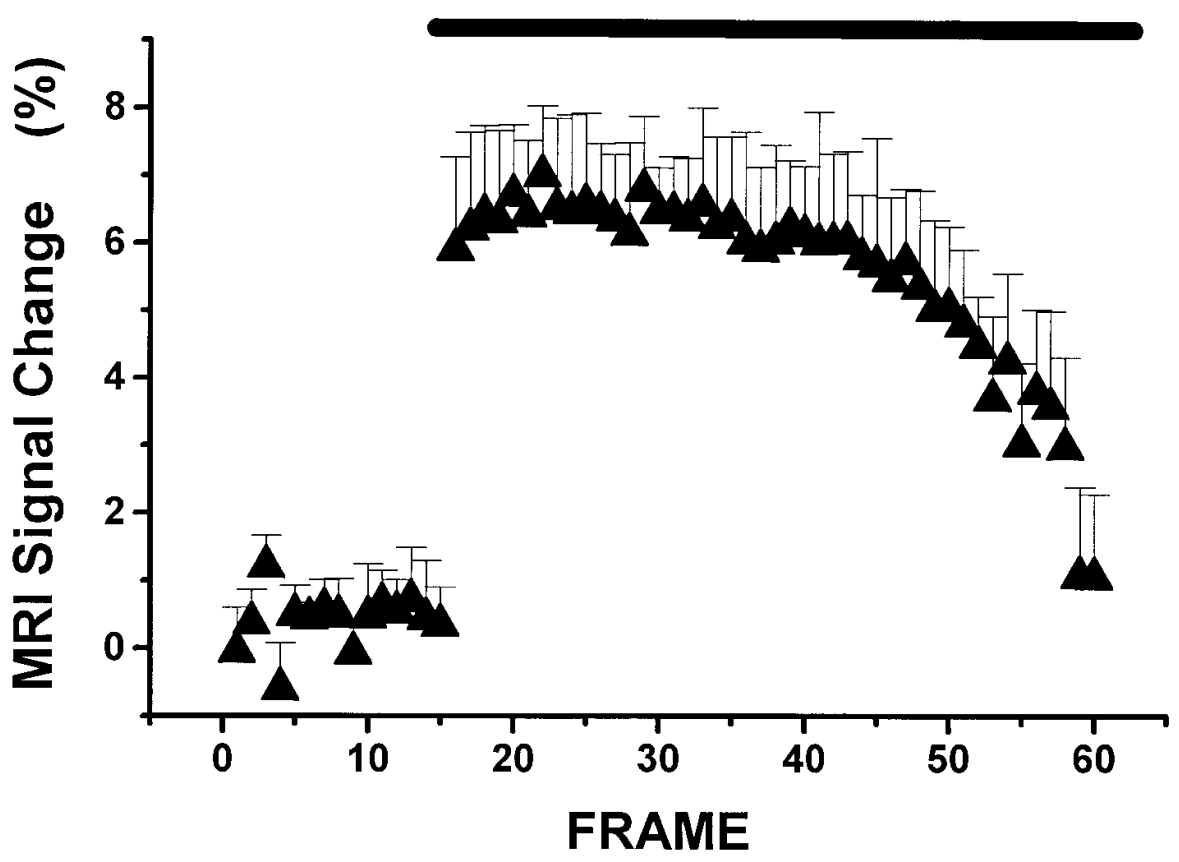

Figure 5. Mean $( \pm \mathrm{SD})$ frame-by-frame fMRI signal changes in eight subjects in the ventral medulla during sustained application of a $30 \mathrm{cmH}_{2} \mathrm{O}$ expiratory load. Note beginning of slow signal decay to values within baseline range within $\sim 28-30$ frames $(285 \mathrm{~s})$ of stimulus application. level-dependent contrast imaging techniques are based on the paramagnetic properties of deoxyhemoglobin, such that a blood vessel containing deoxyhemoglobin placed in a magnetic field will alter the magnetic field in its vicinity (17). Distortion of the magnetic field surrounding venous capillaries by deoxyhemoglobin affects magnetic resonance images of nearby abundant water protons, leading to several thousand-fold signal amplification, thus facilitating measurements (17).

Imaging strategies based on regional oxy-deoxyhemoglobin discrepancies induced by neural recruitment, used in conjunction with specific sensory and motor paradigms, provided for detailed, noninvasive delineation of functional topographic arrangements of cortical regions in the human (6-8, 17, 19-22). Recent technical improvements have allowed considerable enhancement of signal-to-noise ratios, such that signal changes as large as $20 \%$ may be obtained during motor task activation (19-22). However, magnetic field strength (23), white matter density of imaged structures (24), presence of large blood vessels (25), involuntary motion artifacts, and proximity to airbone surfaces may all affect the inherent variability of fMRI signal. Despite such potential limitations, brain regions as localized as the spinal cord and lateral geniculate nucleus have been imaged successfully during specific sensorimotor tasks $(26,27)$. Furthermore, simple motor tasks, such as finger tapping, elicit fMRI signal changes up to $10 \mathrm{SD}$ above the intrinsic variability of the baseline signal (28). On the other hand, it is possible that the area undergoing activation following a particular paradigm may be smaller than the region demonstrating fMRI signal increases, since tissue served by the venous capillary draining network may extend beyond the neuronal population undergoing activation. Although cautious inferences from the current results to the extent of activation are appropriate, locations demonstrating significant fMRI signal change in this study should be viewed as those brain structures most likely underlying the response to expiratory resistive loads.

The findings in three subjects undergoing graded expiratory load challenges suggest that a sensitivity threshold is inherent to the imaging strategy, since loads as small as 0.5
$\mathrm{cmH}_{2} \mathrm{O} \cdot$ liter $^{-1} \mathrm{~s}$ can be easily detected by conscious humans (29). It is possible that only loads which require substantial respiratory system recruitment will elicit sufficiently enhanced regional brain blood flow alterations to allow recognition by these MR parameters. Similar load-dependent increases in dyspnea sensation have been reported for increasing expiratory loads and appear related to the magnitude of respiratory muscle output required to sustain a specific target ventilation (30). The dose dependency of the response suggests that further improvements in signal to noise ratios may eventually reveal regional neural activation to smaller loads.

Certain locations which increased regional activity during expiratory loads coincide with those identified recently in similar experiments using inspiratory resistive loads (11). However, several differences between the results obtained with these two stimuli should be emphasized. First, expiratory loads were associated with activation of discrete medullary regions which were not identified during inspiratory loads. In addition, although it is difficult to compare among different sets of images involving different experimental subjects with confidence, the magnitude and pattern of MRI signal change differences for the two stimulation paradigms suggest that closely apposed neuronal populations within brain structures may subserve functionally different tasks. Second, a homogeneous pattern of very rapid signal decay to baseline values was observed at all activated sites after removal of the expiratory resistor. In contrast, after inspiratory loads were discontinued, some brain regions, such as the ventral and dorsal pons and basal forebrain, showed slow fMRI signal decreases over time; while in others, immediate signal returns to baseline, similar to those observed in this study, occurred (11). The significance of such variations in off-stimulus signal kinetics is unclear; they may represent brain regions playing a role in short-term potentiation or system inertia mechanisms of respiration, which may be preferentially activated by inspiratory, rather than expiratory, loads (31). Third, we observed reduced signal increases within activated sites with subsequent expiratory and inspiratory load applications. The mechanisms mediating such reductions in MR 
signal are unknown and raise the possibility that either rapid load habituation or neural learning processes might lead to reduced numbers of recruited neurons to achieve similar effector responses (32).

The possibility that habituation may be operative during our studies is further suggested by the observation that MR signal decreased over time with sustained stimulation (Fig. 5). Indeed, Haxhiu and colleagues (33) demonstrated that the prolongation in expiratory duration associated with an expiratory resistive load gradually returned to control levels when the load was maintained. However, another potential explanation for the gradual MR signal decline may reside in alterations in cellular oxygen extraction and utilization developing during sustained stimulation as well as from improved vasomotor flow regulation. A gradual transition of activated neurons from nonoxidative to oxidative energy pathways would be expected to occur with sustained stimulation (16), and such an increase in cellular aerobic metabolism would lead to a gradual increase in venous capillary deoxyhemoglobin concentrations with concomitant MR signal decreases. Such an effect would further be accentuated by blood flow returning to metabolically matched, rather than profuse, supply. Indeed, we showed recently that prolonged photic stimulation is associated with an early MR signal increase in the visual cortex which is followed by a progressive signal decrease to control or even subcontrol levels after 2-5 min (34). Similarly, signal decreases were observed within $3 \mathrm{~min}$ of sustained inspiratory loads, indicating that either habituation mechanisms, transition from anaerobic to aerobic cellular metabolic processes, or both become operative over time. Combined studies aiming at relationships between regional blood flow alterations and changes in neural effector outputs during sustained stimulus applications will be critical to decipher the mechanism(s) underling fMRI signal reductions over time.

Both vagal and diaphragmatic afferents appear to play a crucial function in the integrative response to expiratory loads (35). In contrast, upper airway mechanoreceptors and corresponding afferent projections are not important contributors to the ventilatory response (36). It should also be emphasized that marked differences in ribcage coordination and ventilatory strategy are observed when expiratory loads are applied to conscious versus anesthetized humans, suggesting that cortical or more rostral mechanisms are operative, and greatly modify respiratory compensation to obstructive loads (37). However, we did not examine cortical structures in this study.

The vagus has major synaptic relays in pontomedullary structures which heavily project to the cerebellum, basal forebrain, hypothalamus, and cortex (for comprehensive review see reference 38 ). Thus, activation of midsagittal regions, such as those observed in this study, was anticipated. Afferent activity originating from the lung and thoracic walls in phase with respiration has been recorded in the dorsal columns and dorsal spinocerebellar tract in the cat, and direct collaterals to both dorsal and ventral respiratory groups are also apparent (39). The dorsal column recruitment may underlie the gracile and cuneate activation observed here. Stimulation of the cervical vagus evokes responses in the nucleus tractus solitarius, ventral respiratory group, and dorsal respiratory group (40), as well as in the parabrachial and Kölliker-Fuse nuclei of the pons (41), raphe magnus, and locus coeruleus (42). Diencephalic regions, such as the ventrolateral thalamic nuclei, receive major inputs from the cerebellum (43), and respiratory-related neural activity has been recorded from hypothalamic and other rostral brain sites (44). Stimulation of certain cerebellar nuclei exerts prominent effects on respiration; the vermis (45, 46), fastigial (47), infracerebellar, and anterior interposed nuclei $(48,49)$ all play significant roles in modulating vagal inputs during unloaded and loaded expiration. In humans, both PET studies of volitional expiration (5) and current studies using expiratory loads support the concept that cerebellar structures are an integral part of the response loop to moment-to-moment spontaneous or imposed alterations in lung, airway, and chest wall loads.

In conclusion, we demonstrate in awake healthy humans the location of activated brain sites contained within a midsagittal plane during application of resistive expiratory loads. The MRI signal changes elicited by this stimulus paradigm display temporal and magnitude dependencies and emphasize both the nonstationary characteristics and potential pitfalls of functional brain MR imaging.

\section{Acknowledgments}

This study was supported by National Institute of Child Health and Human Development grants HD-22506 and HD-22695 (R. M. Harper) and HD-01072 (D. Gozal).

This work served in partial fulfillment of the requirements for an M.D. Thesis from the Faculté de Médecine de Rouen, France (O. Omidvar).

\section{References}

1. Turcotte, H., M. Tahan, P. Leblanc, and L. P. Boulet. 1993. Perception of acute or progressive resistive loads in normal and asthmatic subjects. Respiration. 60:203-211.

2. Kiruchi, Y., S. Okabe, G. Tamura, W. Hida, M. Homma, K. Shirato, and T. Takishima. 1994. Chemosensitivity and perception of dyspnea in patients with a history of near-fatal asthma. N. Engl. J. Med. 330:1329-1334.

3. Altose, M. D., and N. S. Cherniack. 1981. Respiratory sensation and respiratory muscle activity. Adv. Physiol. Sci. 10:111-119.

4. Davenport, P. W., W. A. Freedman, F. J. Thompson, and O. Franzen. 1988. Respiratory-related cortical potentials evoked by inspiratory occlusion in humans. J. Appl. Physiol. 60:1843-1848.

5. Ramsay, S. C., L. Adams, K. Murphy, D. R. Corfield, S. Grootoonk, D. L. Bailey, R. S. J. Frackowiak, and A. Guz. 1993. Regional cerebral blood flow during volitional expiration in man: a comparison with volitional inspiration. J. Physiol. (Lond.). 461:85-101.

6. Ogawa, S., D. W. Tank, R. Menon, J. M. Ellerman, S. G. Kim, H. Merkle, and K. Ugurbil. 1992. Intrinsic signal changes accompanying sensory stimulation: functional brain mapping with magnetic resonance imaging. Proc. Natl. Acad. Sci. (USA). 89:5951-5955.

7. McCarthy, G., A. M. Blamire, D. L. Rothman, R. Gruetter, and R. G. Shulman. 1993. Echo-planar magnetic resonance imaging studies of frontal cortex activation during word generation in humans. Proc. Natl. Acad. Sci. (USA). 90:4952-4956.

8. Kwong, K. K., J. W. Belliveau, D. A. Chesler, I. E. Goldberg, R. M Weisskoff, B. P. Poncelet, B. N. Kennedy, B. E. Hoppel, M. S. Cohen, R. Turner, et al. 1992. Dynamic magnetic resonance imaging of human brain activity during primary sensory stimulation. Proc. Natl. Acad. Sci. (USA). 89:56755679.

9. Frahm, J., H. Bruhn, K. D. Merboldt, and W. Hanicke. 1992. Dynamic MR imaging of human brain oxygenation during rest and photic stimulation. $J$. Magnet. Reson. Imag. 2:501-505.

10. Gozal, D., G. M. Hathout, K. A. T. Kirlew, H. Tang, M. S. Woo, J. Zhang, R. B. Lufkin, and R. M. Harper. 1994. Localization of putative neural respiratory regions in the human by functional magnetic resonance imaging. $J$. Appl. Physiol. 76:2076-2083.

11. Gozal, D., O. Omidvar, K. A. T. Kirlew, G. M. Hathout, R. Hamilton, J. Zhang, R. B. Lufkin, and R. M. Harper. 1995. Identification of human brain regions underlying responses to inspiratory loading with functional magnetic resonance imaging. Proc. Natl. Acad. Sci. (USA). 92:6607-6611.

12. Fox, P. T., M. A. Mintun, M. E. Raichle, F. M. Miezin, J. M. Allman, and D. C. Van Essen. 1986. Mapping of human visual cortex with positron emission tomography. Nature (Lond.). 323:806-809.

13. Fox, P. T., and M. E. Raichle. 1986. Focal physiological uncoupling of 
cerebral blood flow and oxidative metabolism during somatosensory stimulation in human subjects. Proc. Natl. Acad. Sci. (USA). 83:1140-1144.

14. Phelps, M. E., D. E. Kuhl, and J. C. Mazziotta. 1981. Metabolic mapping of the brains' response to visual stimulation: studies in humans. Science (Wash. DC). 211:1445-1448

15. Fox, P. T., M. E. Raichle, M. A. Mintun, and C. Dence. 1988. Nonoxidative glucose consumption during focal physiology neural activity. Science (Wash. DC). 241:462-464.

16. Prichard, J., D. Rothman, E. Novotny, O. Petroff, T. Kuwabara, M. Avison, A. Howseman, C. Hanstock, and R. Shulman. 1991. Lactate rise detected by ${ }^{1} \mathrm{H}$ NMR in human visual cortex during physiologic stimulation. Proc. Natl. Acad. Sci. (USA). 88:5829-5831.

17. Ogawa, S., T. M. Lee, A. S. Nayak, and P. Glynn. 1990. Oxygenationsensitive contrast in magnetic resonance image of rodent brain at high magnetic fields. Magn. Reson. Med. 14:68-78.

18. Hoshi, Y., and M. Tamura. 1993. Dynamic multichannel near-infrared optical imaging of human brain activity. J. Appl. Physiol. 75:1842-1846.

19. Tootell, R. B. H., J. B. Reppas, K. K. Kwong, R. Malach, R. T. Born, T. J. Brady, B. R. Rosen, and J. W. Belliveau. 1995. Functional analysis of human MT and related visual cortical areas using magnetic resonance imaging. $J$. Neurosci. 15:3215-3230.

20. Connelly, A., G. D. Jackson, R. S. Frackowiak, J. W. Belliveau, F. Vargha-Khadem, and D. G. Gadian. 1993. Functional mapping of activated human primary cortex with a clinical MR imaging system. Radiology. 188:125-130.

21. Schad, L. R., U. Trost, M. V. Knopp, M. Muller, and W. J. Lorenz. 1993. Motor cortex stimulation measured by magnetic resonance imaging on a standard 1.5T clinical scanner. Magn. Reson. Imaging. 11:461-464.

22. Fried, I., D. Gozal, K. A. T. Kirlew, G. M. Hathout, H. Tang, J. X. Zhang, and R. M. Harper. 1994. Dynamic magnetic resonance imaging of human Rolandic cortex. Neuroreport. 5:1593-1596.

23. Bandettini, P. A., E. C. Wong, A. Jesmanowicz, R. Prost, R. W. Cox, R. S. Hinks, and J. S. Hyde. 1994. MRI of human brain activation at 0.5 T, 1.5 T, and 3.0 T: comparisons of R2* and functional contrast to noise ratio. Proc. Soc. Magn. Reson. 1:434a. (Abstr.)

24. Turner, R., D. Le Bihan, C. T. W. Moonen, D. Despres, and J. Frank. 1991. Echo-planar time course MRI of cat brain oxygenation changes. Magn. Reson. Med. 22:159-166.

25. Frahm, J., K. D. Merboldt, W. Hanicke, A. Kleinschmidt, and H. Boecker. 1994. Brain or vein-oxygenation or flow? On signal physiology in functional MRI of human brain activation. NMR Biomed. 7:45-53.

26. Yoshizawa, T., J. A. Sanders, G. J. Moore, M. D. McKay, T. Nose, and L. O. Sillerud. 1993. Functional MRI of spinal cord activity during a motor task. Proc. Soc. Magn. Reson. 1:55a. (Abstr.)

27. Frahm, J., K. D. Merboldt, W. Hanicke, A. Kleinschmidt, and H. Steinmetz. 1993. High-resolution functional MRI of focal subcortical activity in the human brain. Long-echo time FLASH of the lateral geniculate nucleus during visual stimulation. Proc. Soc. Magn. Reson. 1:57a. (Abstr.)

28. DeYoe, E. A., P. Bandettini, J. Neitz, D. Miller, and P. Winans. 1994. Functional magnetic resonance imaging (FMRI) of the human brain. J. Neurosci. Methods. 54:171-187.

29. Puddy, A., G. Giesbrecht, R. Sanii, and M. Younes. 1992. Mechanism of detection of resistive loads in conscious humans. J. Appl. Physiol. 72:2267-2270.

30. Chonan, T., M. D. Altose, and N. S. Cherniack. 1990. Effects of expiratory resistive loading on the sensation of dyspnea. J. Appl. Physiol. 69:91-95.

31. Leevers, A. M., P. M. Simon, L. Xi, and J. A. Dempsey. 1993. Apnea following normocapnic mechanical ventilation in awake mammals: a demonstration of control system inertia. J. Physiol. (Lond.). 472:749-768.

32. Jenkins, I. H., D. J. Brooks, P. D. Nixon, R. S. Frackowiak, and R. E.
Passingham. 1994. Motor sequence learning: a study with positron emission tomography. J. Neurosci. 14:3775-3790.

33. Haxhiu, M. A., E. Van Lunteren, and N. S. Cherniack. 1989. Responses of upper-airway dilating muscles and diaphragm activity to end expiratory pressure loading in anesthetized dogs. Respiration. 56:1-10.

34. Hathout, G. M., K. A. T. Kirlew, G. J. K. So, D. R. Hamilton, J. X Zhang, U. Sinha, S. Sinha, J. Sayre, D. Gozal, R. M. Harper, and R. B. Lufkin. 1994. MR imaging signal response to sustained stimulation in human visual cortex. J. Magn. Reson. Imag. 4:537-543.

35. Barriere, J. R., S. Delpierre, M. J. Del Volgo, and Y. Jammes. 1993. Comparisons among external resistive loading, drug-induced bronchospasm, and dense gas breathing in cats: roles of vagal and spinal afferents. Lung. 171: $125-136$.

36. O'Donnell, D. E., R. Sanii, and M. Younes. 1988. External mechanical loading in conscious humans: role of upper airway mechanoreceptors. J. Appl. Physiol. 65:541-548.

37. Isono, S., T. Nishino, K. Sugimori, and T. Mizuguchi. 1990. Respiratory effects of expiratory flow-resistive loading in conscious and anesthetized humans. Anesth. Analg. 70:594-599.

38. Kubin, L., and R. O. Davies. 1995. Central pathways of pulmonary and airway vagal afferents. In Regulation of Breathing. Lung Biology in Health and Disease. J. A. Dempsey and A. I. Pack, editors. Marcel Dekker, Inc., New York. 219-284.

39. Shannon, R., S. Saporta, and B. G. Lindsey. 1982. Transmission of intercostal muscle proprioceptor afferent information to medullary respiratory areas. Exp. Neurol. 78:222-225.

40. McCrimmon, D. R., D. F. Speck, and J. L. Feldman. 1987. Role of the ventrolateral region of the nucleus tractus solitarius in processing respiratory afferent input from vagus and superior laryngeal nerves. Exp. Brain Res. 67: 449-459.

41. Cohen, M. I., C. F. Shaw, and R. Barnhardt. 1993. Connectivity of rostral pontine inspiratory modulated neurons as revealed by responses to vagal and superior laryngeal afferent stimulation. In Respiratory Control: Centra and Peripheral Mechanisms. D. F. Speck, M. S. Dekin, W. R. Revelette, and D. T. Frazier, editors. Lexington University Press, Lexington, KY. 91-94.

42. Lucier, G. E., and B. J. Sessle. 1981. Presynaptic excitability changes induced in the solitary tract endings of laryngeal primary afferents by stimulation of nucleus raphe magnus and locus coeruleus. Neurosci. Lett. 26:221-226.

43. Asanuma, C., W. T. Thach, and E. G. Jones. 1983. Distribution of cerebellar terminations and their relation to other afferent terminations in the thalamic ventral lateral region of the monkey. Brain Res. Rev. 5:237-265.

44. Radna, R. J., and P. D. MacLean. 1981. Vagal elicitation of respiratorytype and other unit responses in basal limbic structures of squirrel monkeys. Brain Res. 213:45-61.

45. Perrin, J., and J. Crousillat. 1985. The projection of vagal afferents on the cerebellar vermis of the cat. J. Auton. Nerv. Syst. 13:175-177.

46. Tong, G., L. T. Robertson, and J. Brons. 1991. Vagal and somatic representation by the climbing fiber system in lobule 5 of the cat cerebellum. Brain Res. 552:58-66.

47. Xu, F., and D. T. Frazier. 1994. Cerebellar role in the load-compensating response of expiratory muscle. J. Appl. Physiol. 77:1232-1238.

48. Huang, Q., D. Zhou, and W. M. St. John. 1991. Vestibular and cerebellar modulation of expiratory motor activities in the cat. J. Physiol. (Lond.). 436: $385-404$.

49. Huang, Q., D. Zhou, and W. M. St. John. 1993. Cerebellar control of expiratory activities of medullary neurons and spinal nerves. J. Appl. Physiol. 74: 1934-1940. 